\title{
Gratuidad en la educación superior chilena: análisis de prensa basado en minería de datos ${ }^{*}$ Gratuity in Chilean Higher Education: Press Analysis based on Data Mining
}

\author{
Felipe Nesbet Montecinos \\ Universidad Austral de Chile, Chile \\ felipenesbet@gmail.com \\ Luis Cárcamo Ulloa \\ Universidad Austral de Chile, Chile \\ lcarcamo@uach.cl
}

\section{RESUMEN}

El presente artículo investiga sobre los titulares de los medios de comunicación chilenos referentes a gratuidad en la educación superior, que fue una de las temáticas más contingentes tanto en la agenda política como en la noticiosa, desde que emergió el llamado movimiento estudiantil en 2011 a la fecha. Dichos titulares se obtuvieron de las cuentas en la red Twitter de 290 medios de comunicación chilenos. En total se trabajó con 2137 titulares, recolectados entre el $1^{\circ}$ de marzo y el 31 de julio de 2016. Con base en este material se descubrió cuáles fueron las voces que informaron sobre el tema, los tipos de medios y la calificación de los titulares, lo que realizó un trío de jueces externos.

Palabras clave: gratuidad en la educación, prensa chilena, medios de comunicación, voces, Twitter

\begin{abstract}
This article is a research of the headlines of the Chilean media regarding gratuity in higher education, which was one of the most contingent topics on both the political and news agenda, since the so-called 2011 student movement emerged to the date. These headlines were obtained from the accounts on the Twitter network of 290 Chilean media. In total, we worked with 2137 headlines, collected between March 1 and July 31, 2016. Based on this material, we discovered the voices that reported on the subject, the types of media and the qualification of the headlines, which was carried out by a trio of external judges.
\end{abstract}

Keywords: free education, Chilean press, media, voices, Twitter

\footnotetext{
*El presente artículo se basa en los aportes del Fondo Nacional del Desarrollo Científico y Tecnológico (Fondecyt $N^{\circ}$ 1150545), titulado: "Redes Sociales y Medios de Comunicación: Modelo de análisis basado en minería de datos para la comprensión del ecosistema informativo chileno en internet y la educomunicación ciudadana en la red". Este proyecto es dirigido por el Dr. Luis Cárcamo y asistido por el Mg. Felipe Nesbet. Además, recibió la colaboración y traducción al idioma inglés por la Mg. Kristin Becker.
} 


\section{INTRODUCCIÓN}

Indudablemente, la irrupción del movimiento estudiantil en 2011, que, entre sus principales demandas, planteaba la necesidad de la gratuidad en la educación superior, marcó un importante hito en el desarrollo político-social chileno en la última década. En efecto, esta temática fue una de las más relevantes en las elecciones presidenciales de 2013 y ha estado presente en la gestión de las políticas públicas durante todo el mandato de Michelle Bachelet (2014-2018).

Como es lógico, la prensa tuvo un relevante papel en este proceso informando sobre las múltiples aristas de este fenómeno, presentando (en concordancia con cada línea editorial) las distintas visiones y ejerciendo una influencia sobre el público que recibe la información. Dada la importancia del tema, este artículo presenta como objetivo investigar qué medios y voces tuvieron más titulares sobre la gratuidad en educación, cuáles fueron los más repetidos por los distintos medios de comunicación y qué connotación se le puede atribuir a dichos enunciados. Para evitar que la temática se mezcle con cuestiones electorales, se decidió abarcar un periodo que no coincidiera con uno de estos procesos, por lo que se optó por el primer semestre de 2016. Durante este lapso de tiempo el tema tuvo cierta relevancia, pues fue la primera vez que se ejerció la gratuidad y los estudiantes mantuvieron movilizaciones críticas respecto de los alcances reales de la iniciativa.

\section{EDUCACIÓN SUPERIOR CHILENA: CONTEXTO}

Para comenzar, es necesario contextualizar sobre la educación superior chilena. En 1981, la dictadura militar, liderada por el general Augusto Pinochet, implementó una reforma en el sistema universitario, que implicó la atomización de las dos principales entidades académicas nacionales (Universidad de Chile y Técnica del Estado), y el fin de la gratuidad en la educación superior (Bellei, Cabalin y Orellana, 2014). Por ende, la familia del estudiante o él, tras concluir sus estudios, pagarían su educación universitaria. De esta forma nació el sistema de Crédito Fiscal Universitario, que entregaba este préstamo a los universitarios, de acuerdo con los ingresos de su 
grupo familiar. Dicho crédito debía ser cancelado dos años después de su egreso. La deuda se pagaría en 10 cuotas anuales, que serían calculadas mediante las Unidades Tributarias Mensuales (UTM), con un interés devengado de $1 \%$. El monto cancelado se relacionaría con los ingresos de la persona.

En 1994 el modelo se vuelve a modificar y se crea el Fondo Solidario de Crédito Universitario, que implicó un aumento en la tasa de interés anual $(2 \%)$ y la ampliación del plazo de pago hasta 15 años. Con el propósito de facilitar la presencia de jóvenes de escasos recursos en el sistema de educación superior, el gobierno de Ricardo Lagos creó, en 2005, el Crédito con Aval del Estado (CAE), que implicaba que los estudiantes podían financiar sus estudios mediante créditos con la banca privada, con lo que la tasa de interés de los mismos aumentaba hasta $6 \%$.

La instalación de este modelo de financiamiento, que destruyó el concepto de educación gratuita universitaria y adosó la responsabilidad del financiamiento a los estudiantes (en especial mediante sus familias) no se puede entender disociado de la adopción del modelo neoliberal a mediados de los ańos setenta, siendo Chile el primer país del mundo que adoptó esta doctrina económica (Hobsbawm, 1999; Harvey, 2007). A grandes rasgos, el neoliberalismo propone la disminución del papel económico del Estado como ente regulador y administrador de sectores económicos, y prioriza el rol de las entidades privadas (Harvey, 2007). Por eso, desde la óptica neoliberal, la educación no se concibe como un derecho, sino como un bien de consumo; así lo señaló el propio presidente Sebastián Pińera en el momento álgido de la movilización universitaria (Emol, 2011). En consecuencia, para el neoliberalismo la educación es un instrumento de movilidad social, que permite que las personas puedan desarrollarse económicamente, por lo que no se justifica que el Estado tenga que financiar algo que solamente favorecerá a un individuo para su beneficio personal.

En medio de la dictadura, y con la posterior desmovilización política de la transición en el poder, auspiciada desde los propios sectores democráticos (Moulian, 1997), la discusión sobre la gratuidad universitaria estuvo muy ausente en la agenda política y noticiosa del país. Incluso en la llamada "Revolución Pingüina", que estalló en 
2006 y fue protagonizada por los estudiantes secundarios, el tema fue cubierto por la prensa, pero no logró tener impactos reales en la definición de políticas gubernamentales.

Por otro lado, en un contexto en el que la educación universitaria, en la mayoría de los casos, significaba la obtención de buenos puestos laborales, con el consecuente beneficio económico, el sistema de crédito universitario no provocaba una crítica social generalizada. Además, se debe tomar en cuenta que, en principio, estábamos hablando de un universo muy reducido de estudiantes, que fue creciendo exponencialmente con el paso de los años. En 1980 la matrícula en educación superior llegaba a cerca de 117 mil personas, lo que equivalía a alrededor 7.2\% del grupo etario entre 18 y 24 ańos. En 1990 el número había crecido a 245 mil y para 2007 la cifra era de 678 mil. En efecto, para 2009, la población matriculada en las Universidades chilenas solamente era superada en la Organización para la Cooperación y el Desarrollo (OCDE) por Israel, Irlanda y México (OCDE, 2009).

Con el siempre creciente número de nuevos profesionales para fines de la década de 2000, la empleabilidad de los jóvenes profesionales chilenos se estaba deteriorando tanto en la obtención de empleos como en la calidad de los mismos. No se debe olvidar que en 2008 estalló la crisis económica internacional, que generó un alza de la cesantía en el país, la cual llegó a los dos dígitos. La Encuesta Nacional de la Juventud de ese año reportó que 43.8\% de los jóvenes no trabajaba en un área relacionada con sus estudios, y $21.4 \%$ de los que contaban con estudios universitarios se encontraban cesantes (Instituto Nacional de la Juventud, INJUV, 2010), más que duplicando el promedio nacional.

Por eso, no sería hasta 2011 que la problemática por la gratuidad en la educación superior detonaría; ${ }^{1}$ mientras el gobierno hablaba de que ese periodo sería el "El Año de la Educación Superior". En el estallido de este malestar fue muy sintomático que un estudio de la OCDE pusiera a colación el alto costo de la educación superior

\footnotetext{
${ }^{1} \mathrm{El}$ hecho que las protestas no se hayan producido en 2010, apenas iniciado el nuevo gobierno, responde a dos fenómenos que marcaron la agenda en ese periodo. Primero, el terremoto del 27 de febrero, que asoló varias regiones del país, y segundo, el rescate de los 33 mineros de la Mina de San José, un episodio que no sólo fue seguido atentamente por los chilenos sino por todo el mundo.
} 
chilena. ${ }^{2}$ La investigación señaló que los aranceles chilenos eran superiores a los de Gran Bretańa, Italia, Holanda, Austria, Bélgica, España, y estaban en la misma línea que Australia, Canadá, Japón y Corea del Sur; todos países de un ingreso per cápita muy superior al chileno. En el concierto iberoamericano, Chile era el único país con aranceles de 3000 dólares anuales, mientras el rango siguiente fluctuaba entre 500 y 1000 dólares, que solamente lo ocupaban Costa Rica y Colombia (OCDE, 2009).

Obviamente, la irrupción de esta problemática no se puede desligar de la asunción del primer gobierno derechista en veinte años, liderado por el empresario Sebastián Pińera. Esto generó que los sectores centroizquierdistas, que habían detentado el poder político en las últimas décadas, se unieran a los movimientos sociales en la crítica al modelo neoliberal. El nuevo gobierno carecía de los vínculos con las organizaciones populares, que sí tenían los gobiernos anteriores, lo que jugaba en contra de su posibilidad de controlar las protestas (Tironi, 2011). Es lógico pensar que muchos de los participantes en el llamado movimiento estudiantil (como lo denominó la prensa, aunque confluían ahí distintas organizaciones con diversos objetivos) fueron parte de la Revolución Pingüina y aprendieron de las lecciones de esta experiencia, que fracasó en sus objetivos a largo plazo (Cabalin, 2012, y Bellei, Cabalin y Orellana, 2014). Para 2011, los estudiantes contaban con líderes carismáticos que alcanzaron fama internacional, como Camila Vallejo, Karol Cariola, Giorgio Jackson y Gabriel Boric, quienes posteriormente pasaron a integrar el Parlamento. Este movimiento alcanzó altos niveles de popularidad incluso entre las clases medias, que habían respaldado electoralmente a Piñera, a quienes la demanda por educación gratuita les atañía íntimamente (Organización de las Naciones Unidas para la Infancia, UNICEF, 2014). Las protestas quebraron la concepción, que primaba desde el regreso a la democracia, que caracterizaba a la juventud chilena con la apatía y el desinterés político (Scherman, Arriagada y Valenzuela, 2012).

\footnotetext{
${ }^{2} \mathrm{La}$ OCDE es una entidad que reúne a los países más desarrollados del mundo, por lo que el gobierno chileno, tanto el centroizquierdista de Michelle Bachelet como el derechista que lo sucedió, celebraron profusamente el ingreso a esta organización (como el primer país sudamericano y el segundo país iberoamericano en integrarse, después de México).
} 
En síntesis, como lo planteó el sociólogo Juan Carlos Gómez, las movilizaciones estudiantiles de 2011 (a las que se sumaron las protestas por la instalación de represas en la Patagonia) significaron la segunda coyuntura crítica que vivió el sistema neoliberal en Chile, tras las protestas sociales por la crisis económica de 1982 (Gómez, 2012).

\section{GRATUIDAD EN LAS ELECCIONES PRESIDENCIALES}

Aunque el movimiento estudiantil no pudo mantener la gran presencia mediática y social en los años posteriores, sí dejó instalada en las elecciones presidenciales de 2013 el problema educacional, que fue uno de los principales tópicos en la agenda electoral. En efecto, salvo la candidata oficialista de derecha, Evelyn Matthei (2013), los siete restantes incluían la educación gratuita en sus programas de gobierno o propuestas programáticas. Roxana Miranda (2013), Marcel Claude (2013) y Alfredo Sfeir (2013) planteaban incluir este derecho de forma universal en todos los niveles. Marco EnríquezOminami (2013), candidato del Partido Progresista (PRO), hablaba de gratuidad en las universidades estatales; lo mismo planteaba el independiente Franco Parisi (2013), incluyendo aranceles diferenciados (que se establecían mediante el quintil socioeconómico al que perteneciera la familia del estudiante). El exdiputado demócratacristiano, Tomás Jocelyn-Holt (2013), establecía avanzar en la gratuidad para cuatro quintas partes de la población, y fijar aranceles que no subieran todos los años. En la misma línea, Ricardo Israel (2013), abanderado del Partido Regionalista Independiente (PRI), proponía que este beneficio llegara a ciertos quintiles de la población. ${ }^{3}$ Pese a haber declarado no estar de acuerdo con la educación universitaria gratuita universal (Cooperativa, 2013), el programa de Michelle Bachelet, quien a la postre obtendría la presidencia, señalaba plazos para alcanzar dicha meta:

\footnotetext{
${ }^{3}$ En el programa de este candidato se señala que entre el $10^{\circ}$ y el $8^{\circ}$ se establecerá un arancel diferenciado, y entre el $7^{\circ}$ y el $1^{\circ}$, que son los grupos de mayores ingresos, podrán acceder a educación gratuita. Se estima que esto respondió a una confusión en la redacción del documento por parte del equipo de Israel.
} 
Avanzaremos gradualmente en la gratuidad universal y efectiva de la educación superior, en un proceso que tomará seis años. Durante el próximo periodo de Gobierno, accederán a la gratuidad al menos los y las estudiantes pertenecientes al $70 \%$ más vulnerable de la población, abarcando extensamente a la clase media (Bachelet, 2013, p. 14).

Para efectos comparativos, en las elecciones presidenciales de 2009 solamente Jorge Arrate, ${ }^{4}$ candidato respaldado por el Partido Comunista y otras entidades de izquierda, habló de garantizar la educación gratuita a los nińos y jóvenes en un plazo breve, que no se detallaba. No obstante, lo anterior se contradice con su planteamiento de terminar con "el sistema de crédito actual a los estudiantes, reemplazándolo por asignaciones presupuestarias directas de matrículas y aranceles para todos los alumnos que lo necesiten" (Arrate, 2009, p. 39).

El programa de Marco Enríquez-Ominami se basaba en los conceptos de calidad y equidad, abarcando escasamente la educación superior. ${ }^{5}$ De hecho, en este aspecto solamente se mencionan premios de excelencia en este nivel educativo (Enríquez-Ominami, 2009).

Los dos candidatos que pasarían a segunda vuelta (Sebastián Piñera y Eduardo Frei) basaban sus propuestas en la mejora del acceso a la educación superior mediante becas. Frei (2009) ofrecía becas completas a los estudiantes de $1^{\circ}$ y $2^{\circ}$ quintiles que estuvieran dentro del 20\% de los mejores resultados de sus cursos. Mientras Piñera (2009, p. 89), quien resultaría ganador en los comicios, solamente hablaba de perfeccionar el sistema de becas, "terminando con la discriminación en el financiamiento según la naturaleza jurídica de la institución de educación superior elegida por el alumno. Se eliminarán gradualmente las diferencias en los sistemas de créditos”.

\footnotetext{
${ }^{4}$ En 1994, Arrate, en su calidad de ministro de educación, fue el gestor de la ampliación del sistema de copago a los colegios públicos. Este mecanismo consistía en el aporte familiar en el financiamiento de los colegios. Aunque esta medida nació de forma voluntaria, en la práctica terminó siendo una obligatoriedad para los estudiantes. La eliminación del copago fue una de las banderas de lucha del movimiento estudiantil, que finalmente fue derogado por el segundo gobierno de Michelle Bachelet.

${ }^{5}$ Es llamativo que en su programa se señale que su gobierno no prohibirá el lucro en la educación, lo que estaba (y está) sancionado por la Ley, pero con base en resquicios.
} 


\section{ANÁLISIS DE MEDIOS}

Aunque el movimiento estudiantil chileno ha sido ampliamente estudiado por distintos autores que lo abordaron desde disímiles miradas, entre ellas también se incluyó el tratamiento mediático. Tal vez uno de los trabajos más completos que investigaron el tema fue el estudio de Cabalin (2013) acerca del análisis de las editoriales de los medios escritos sobre el movimiento estudiantil. Desde una perspectiva regional, Browne, Monsalve y Romero (2015) estudiaron la cobertura del movimiento estudiantil en la Región de Los Ríos (Sur de Chile), tanto en la prensa escrita como en la digital. También cabe consignar el trabajo de Romero (2013), que realizó un análisis crítico del tratamiento informativo hacia el movimiento estudiantil y la figura de Camila Vallejo, por parte del periódico Las Últimas Noticias, uno de los de mayor tiraje en el país y caracterizado por su línea sensacionalista.

No obstante, no existe un estudio que analice los titulares de la prensa con una mirada cuantitativa, observando los medios y actores (tanto personas como entidades) que más titulares presentaron sobre el tema, lo que constituye el objetivo que propone esta investigación. Además, el periodo de estudio del presente trabajo comprende un periodo más actual, centrando el análisis en una de las medidas concretas que ofreció el segundo gobierno de Michelle Bachelet como respuesta a las movilizaciones ocurridas entre 2011 y 2013.

El único caso que podría semejarse a nuestro trabajo es la investigación realizada por Cabalin y Antezana (2016) sobre las portadas del periódico Las Últimas Noticias referentes a la política educacional de la administración Bachelet. Aunque este estudió analizó algunos titulares sobre la gratuidad en la educación (4), su cobertura es mucho menor que la de esta investigación, dado que incluyó un solo medio, mientras este trabajo trata de abarcar al grueso de los medios de comunicación chilenos. 


\section{METODOLOGÍA}

\section{Minería de datos}

Esta investigación se realizó siguiendo la metodología basada en minería de datos textuales (Baeza-Yates, 2009), mediante un crawler $a d-h o c^{6}$ para la prensa chilena. Se trata de un software automatizado y propio del presente proyecto de investigación, que recolecta metódicamente los titulares organizados como datos textuales y contabilizando frecuencias de emisión en 290 medios chilenos, mediante sus cuentas de Twitter, almacenando sus titulares en un servidor. Actualmente, la minería de datos es una herramienta bastante extendida para analizar sesgos en la prensa (Saéz-Trumper, Castillo y Lalmas, 2013) o las opiniones en campañas electorales (Best y Meng, 2015). Sobre los datos colectados se instalaron herramientas informáticas tales como Elasticsearch y Kibana, que exploran los datos textuales organizados en una base de datos, para realizar el análisis de estadística descriptiva, sometiendo a juicio de arbitraje un corpus de titulares para evaluar su polaridad; es decir, calificándoles de acuerdo con los criterios básicos de positiva, neutra o negativa.

\section{Corpus}

Para ejecutar este proyecto se optó por el periodo comprendido entre el $1^{\circ}$ de marzo y el 31 de julio de 2016, lo que abarca un semestre académico regular en Chile. La decisión de seleccionar este periodo responde, esencialmente, a que no existe una dedicación noticiosa hacia temas electorales que, eventualmente, mezclarían las discusiones con otros aspectos.

La extracción de datos arrojó una cifra de 1179847 titulares de noticias en 290 cuentas oficiales de medios de comunicación chilenos en Twitter. Para hacer foco en nuestro tópico de investigación filtramos mediante la búsqueda de los conceptos "Reforma educa-

\footnotetext{
${ }^{6}$ Un crawler o araña web es una programación automática que permite que un servidor simule la acción de visitar y guardar la información de muchas páginas web en forma sistemática y paralela. Obviamente, el crawler, va más allá de las posibilidades humanas, ya que recorre 290 medios las 24 horas y los 365 días del año.
} 
cional", "reforma educativa", "gratuidad universitaria", "gratuidad en la educación" y "gratuidad" arrojando una cifra de 3043 y, tras un proceso de chequeo manual de las noticias, para cerciorarnos que hicieran referencia a la gratuidad en la educación superior, se llegó al número final de 2137 titulares. Esto significa 0.18\% de emisiones totales de los medios de comunicación seguidos. Para efectos comparativos, la temática "femicidio", "violencia contra la mujer" y "acoso", un tema que también ha ganado notoriedad en la agenda noticiosa, tuvo una cantidad un poco superior (2 168 menciones). Mientras el concepto AFP (Administradora de fondos de pensiones), sumado a "pensiones" y "jubilación” obtuvo más del doble de titulares, llegando a 5004, lo que significa $0.42 \%$ del total. Mayor aún fue la presencia del tópico "robo", "asalto", "delincuencia", que contó con 6488 menciones, representando $0.54 \%$ de los titulares. Dos de los conceptos más conocidos en Chile, como lo son la presidente Bachelet y el club de fútbol más popular, $\mathrm{Colo}^{7}$ tuvieron más de 11 mil menciones (11740 y 11238 , respectivamente), lo que tampoco alcanza $1 \%$.

Con el corpus ya bien definido se procedió a:

- Categorizar según el foco periodístico de cobertura principal: distinguiendo a los medios de acuerdo con su alcance nacional, regional e hiperlocal (López, Negreira y Rodríguez, 2016). Esta acción busca averiguar qué tipos de medios se refirieron en mayor medida a la gratuidad en educación superior.

- Siguiendo una metodología de análisis de contenidos (Krippendorff, 2004) se identificó a los actores principales mencionados en los titulares de las noticias.

- El tercer procedimiento empleado tiene como finalidad analizar la concentración de tópicos, pues es muy natural que los medios "hablen de las mismas noticias"; para resolver este desafío, el equipo de investigación identificó grupos de noticias que referían a un mismo hecho.

${ }^{7}$ Los otros equipos futbolísticos con mayor adhesión popular usan el nombre de Universidades (Chile y Católica), por lo que la búsqueda se puede confundir con las casas de estudios. 
- Finalmente, se identificó la polaridad positiva, neutra o negativa de los diez titulares más repetidos. En este caso, la metodología solicitó a tres jueces independientes que calificaran la polaridad de 112 titulares de prensa, que se seleccionaron de forma aleatoria, manteniendo la proporción de los medios que más informaron sobre el tema.

\section{RESULTADOS: CARACTERIZACIÓN DEL CORPUS}

En este corpus refinado de 2137 titulares, encontramos 139 medios que realizaron al menos una mención sobre la gratuidad en la educación. Los medios fueron agrupados en tres sectores según el foco periodístico de cobertura principal: nacionales, regionales e hiperlocales (López, Negreira y Rodríguez, 2016).

Nacionales: Medios que cubren hechos noticiosos, preponderantemente, de todo el país (Ejemplo, Radio Cooperativa, La Tercera).

Regionales o Locales: Medios que cubren hechos preponderantemente regionales o provinciales (Ejemplo: La Estrella de Iquique).

Hiperlocales: Medios que cubren hechos de proximidad y responden mayoritariamente a comunas pequeñas del país (Ejemplo: La Voz de Paillaco).

Los resultados nos indican que los medios nacionales son los que tuvieron más titulares sobre el tema, con 1818 enunciados, equivalente a $85 \%$, mientras los medios regionales contaron con 229 titulares, equivalentes a $11 \%$ y los hiperlocales con 96 noticias, representando $4 \%$ del total (ver gráfica 1).

La notable preeminencia de los medios nacionales por sobre los regionales se explica por la mayor capacidad operativa de estas entidades, que cuentan con mayores recursos, tanto humanos como logísticos y financieros, que sus pares regionales e hiperlocales que, la mayoría de las veces, carecen de la capacidad para generar informaciones propias, y se limitan a publicar las noticias que reciben desde los órganos institucionales. 
- Gráfica 1. Tipos de medios con más titulares sobre gratuidad en educación

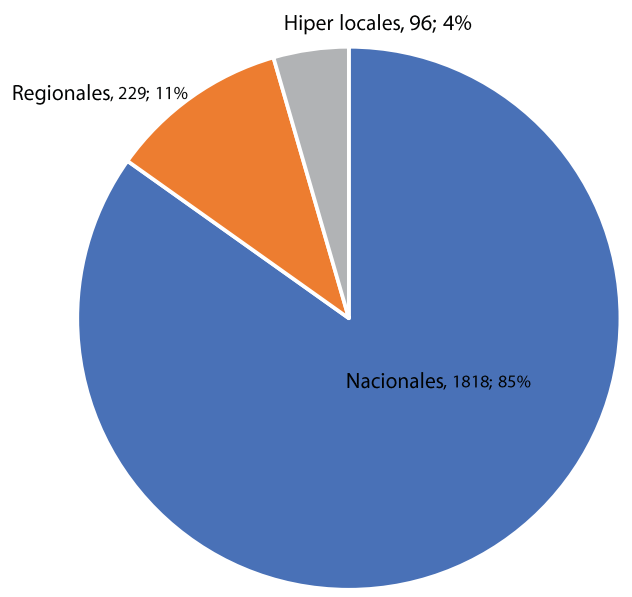

Fuente: elaboración propia.

Diez medios nacionales concentran 53\% de las menciones sobre el tema investigado. En este ítem el medio que más titulares presenta es Emol (portal electrónico de El Mercurio, uno de los periódicos más tradicionales del país), con 216 titulares, que representa 10\%; uno más que La Tercera, otro de los periódicos más importantes del país; el tercer lugar lo ocupa T13 (portal del Canal 13) con 128 titulares, lo que equivale a 6\%; mismo porcentaje para PublimetroChile, que tuvo diez titulares menos que la estación televisiva, y el quinto lugar lo ostenta la Radio Cooperativa con 102 menciones, equivalente a $5 \%$ del total (ver gráfica 2).

Analizando estos datos queda en evidencia que los tres medios que más publicaron sobre el tema están identificados con la derecha política (Emol y La Tercera), mientras T13 es propiedad de Andrónico Luksic, principal fortuna del país. Por otro lado, resulta llamativa la escasa participación del canal 24 Horas TVN, entidad estatal que, en teoría, se esperaría que informara más sobre la principal promesa del gobierno de turno. ${ }^{8}$

\footnotetext{
${ }^{8}$ Aunque el directorio de Televisión Nacional de Chile (TVN) está compuesto por personalidades de todos los conglomerados políticos, en la práctica mantiene una línea editorial favorable a la administración vigente.
} 


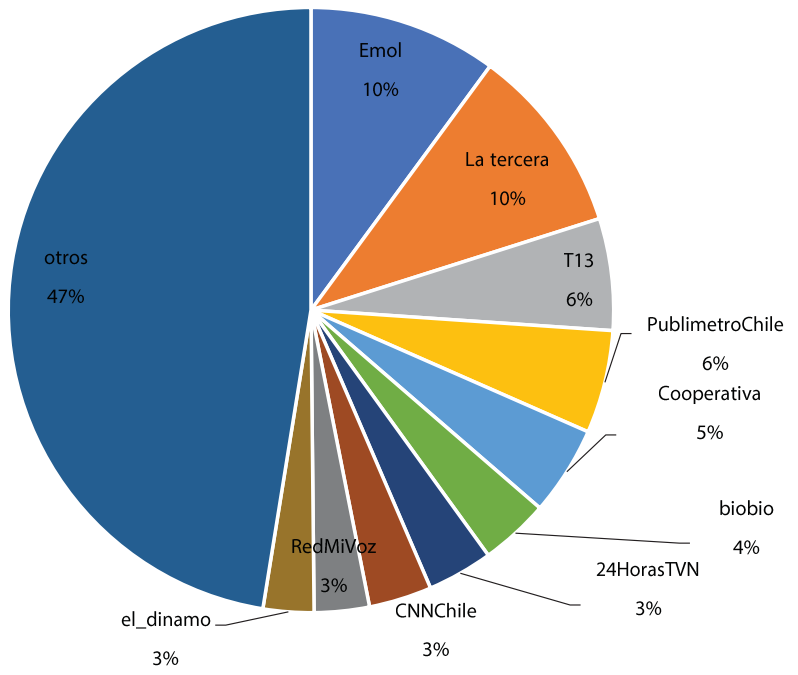

Fuente: elaboración propia.

\section{Actores de la noticia}

En este estudio se detectaron 188 voces que presentaron titulares sobre el tema, de los cuales 136 son personas, 41 instituciones y 11 colectivos (por ejemplo, estudiantes o magallánicos). El actor más mencionado fue el Ministerio de Educación, con 436 titulares, seguido por la ministra de esta cartera, Adriana Delpiano, con 302; el tercer lugar lo ocupó la Confederación de Estudiantes de Chile (Confech), con 151 menciones (ver gráfica 3).

En el análisis de las voces de los titulares destaca el peso de las entidades oficialistas, que representan casi la mitad de las diez voces con más titulares (Mineduc, Adriana Delpiano, Michelle Bachelet, Rodrigo Valdés y Gobierno). En total, 51.9\% de las noticias responden a actores o entidades de gobierno. ${ }^{9}$ Sin embargo, llama la atención el escaso protagonismo de la presidenta Michelle Bachelet, el cuarto lugar en las menciones, con 120 titulares (apenas diez más

\footnotetext{
${ }^{9}$ En este conteo no se incluyó a Erwin Rosas, quien aparece mencionado por un cuestionamiento personal ligado al tema, no por su opinión hacia la gratuidad en la educación.
} 
que su ministro de Hacienda, Rodrigo Valdés, que ocupa el quinto lugar). Lo anterior indica que la ministra de educación, Adriana Delpiano, tomó la vocería del gobierno en desmedro de la propia presidenta, lo que resulta sumamente curioso, tomando en cuenta que estamos hablando de la principal promesa de campaña del gobierno. Obviamente, esto responde a un diseño comunicacional decidido en las más altas esferas de la administración pública. Los porqués de esta decisión dan para un sinfín de conjeturas políticas que escapan a nuestro estudio.

- Gráfica 3. Voces sobre gratuidad en educación

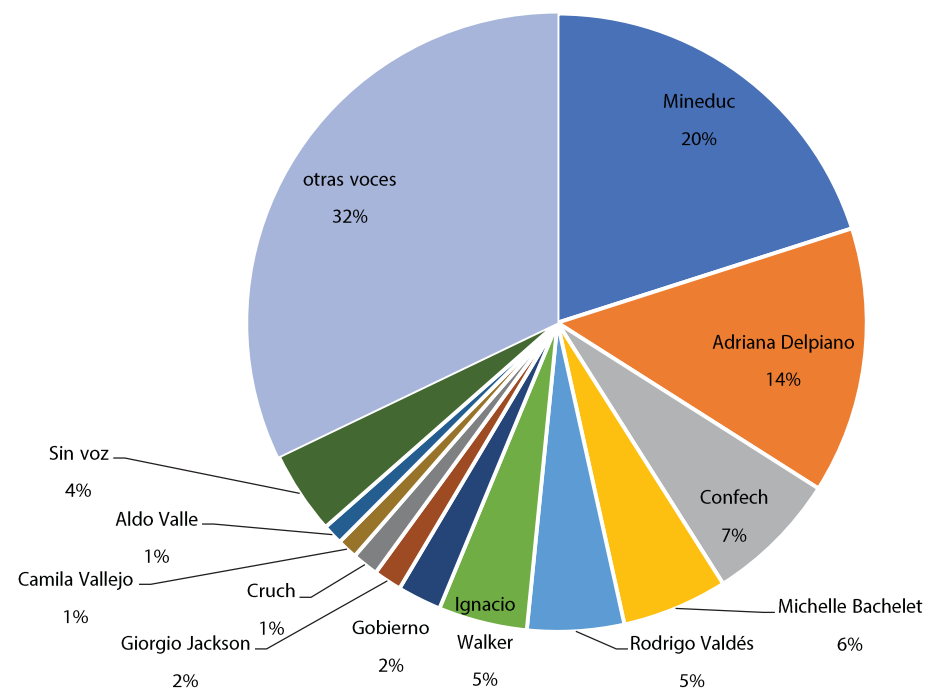

Fuente: elaboración propia.

Frente a la preeminencia de los representantes públicos es interesante observar la escasa representación de los rectores y autoridades de las universidades chilenas, uno de los actores fundamentales en este proceso, quienes, en su conjunto, suman $6.9 \%$ de los titulares. Aunque la Confech ocupa el tercer lugar entre los actores más mencionados, ningún líder estudiantil ni federación tiene una presencia relevante. Las agrupaciones estudiantiles de las Universidades de Chile y la Católica apenas tienen tres y dos titulares respectivamente; las federaciones de estudiantes de la Universidad de Santiago y la 
Universidad Austral tienen un poco más de menciones con siete y seis titulares. Un poco más presenta Camila Rojas, presidenta de la Federación de Estudiantes de la Universidad de Chile (Fech), quien cuenta con diez titulares, mientras la vicepresidenta, Javiera Reyes, tuvo nueve (producto de una toma de la sede de la Junta Nacional de Auxilio y Becas, Junaeb). En comparación, mucho mayor cobertura tuvo la noticia de Clara García, estudiante que fue presentada como caso emblemático de acceso gratuito a la educación superior, que más tarde salió rechazada, quien presentó 20 titulares, o el caso de Erwin Rosas, alto funcionario de un gobierno regional, cuyo hijo fue favorecido por la gratuidad, con 14 menciones por su nombre y nueve con el nombre de su hijo. Lo anterior se puede leer como un intento de los medios por desacreditar la educación gratuita.

\section{ANÁLISIS DE LOS TITULARES}

\section{Análisis de los diez titulares más repetidos}

Como es habitual en la red Twitter, muchos de los titulares son noticias repetidas, por lo que de las 2137 menciones, 671 corresponden a noticias o hechos únicos. De este universo de titulares seleccionamos los diez más repetidos (ver cuadro 1).

Cuadro 1. Titulares más repetidos

\begin{tabular}{|l|c|}
\hline \multicolumn{1}{|c|}{ Noticias } & Repeticiones \\
\hline Asignación de gratuidad por error & 146 \\
\hline Ignacio Walker le cierra la puerta a la gratuidad: "Nos entusiasmamos con metas que son inalcanzables” & 90 \\
\hline Resultados de gratuidad & 87 \\
\hline Delpiano comenta que no se renunciará hasta alcanzar al 70\% más vulnerable & 69 \\
\hline Cómo apelar a gratuidad & 48 \\
\hline Confech tilda de inaceptable la disminución de beneficiados con gratuidad & 46 \\
\hline Ministra de Educación dice que U. Autónoma se coló en gratuidad & 39 \\
\hline 130 mil estudiantes con gratuidad & 36 \\
\hline Beca de alimentación para los beneficiados con gratuidad & 24 \\
\hline Últimos plazos para apelar a gratuidad & 23 \\
\hline
\end{tabular}


En una primera lectura podemos visualizar que cuatro noticias son "vocerías" de personas (Ignacio Walker, Ministra de Educación y Adriana Delpiano) o entidades (Confech), tres son anuncios referentes a la gratuidad (Últimos plazos para apelar a la gratuidad, resultados de gratuidad y cómo apelar a la gratuidad) y otras tres son informaciones (Asignación de gratuidad por error, $130 \mathrm{mil}$ estudiantes con gratuidad y becas de alimentación para los beneficiados con gratuidad).

Un trío de jueces (compuesto por un estudiante de pedagogía, un estudiante de diseño gráfico y un contador público) calificó los titulares mediante los parámetros positivo, neutro o negativo, respecto a la postura frente a la gratuidad universal (ver cuadro 2).

Cuadro 2. Calificación de los titulares más repetidos

\begin{tabular}{|l|l|}
\hline \multicolumn{1}{|c|}{ Noticias } & Juicios \\
\hline Asignación de gratuidad por error & Negativo \\
\hline Ignacio Walker le cierra la puerta a la gratuidad: “Nos entusiasmamos con metas que son inalcanzables” & Negativo \\
\hline Resultados de gratuidad & Neutro \\
\hline Delpiano comenta que no se renunciará hasta alcanzar al 70\% más vulnerable & Positivo \\
\hline Cómo apelar a gratuidad & Neutro \\
\hline Confech tilda de inaceptable la disminución de beneficiados con gratuidad & Negativo \\
\hline Ministra de Educación dice que U. Autónoma se coló en gratuidad & Negativo \\
\hline 130 mil estudiantes con gratuidad & Positivo \\
\hline Beca de alimentación para los beneficiados con gratuidad & Positivo \\
\hline Últimos plazos para apelar a gratuidad & Negativo \\
\hline
\end{tabular}

Fuente: elaboración propia.

Más allá de esta simple observación, con una preeminencia de la calificación negativa, y el hecho que las dos noticias más repetidas fueron negativas, un análisis más detenido de los números nos da una visión sobre la tendencia de los titulares de la prensa sobre la gratuidad en la educación. La repetición de las negativas alcanzó 344 veces, más que duplicando a la repetición de las positivas, que sumaron 129. Incluso, la repetición de las noticias calificadas como neutras fue mayor (135), pese a tratarse solamente de dos titulares.

\section{Calificación de los titulares}

Del universo de noticias seleccionamos 112 titulares. Siguiendo la proporción de las menciones de los medios sobre el tema, se toma- 
ron 20 noticias de Emol, 20 de La Tercera, 12 de T13, diez de Publimetro y Cooperativa, ocho de Bio, seis de 24 Horas TVN, CNN Chile, Red Mi Voz y El Dinamo, a las que se sumaron las diez noticias más repetidas. Los titulares fueron organizados de forma aleatoria, con tal de evitar la posibilidad que una tendencia se viera repetida. Esta base fue sometida a calificación por nuestros tres jueces, manteniendo las evaluaciones de positivo, neutro o negativo.

El resultado final otorgó 44 titulares negativos, 43 positivas, 15 neutras y diez que fueron sancionadas de distinta forma por cada uno de los jueces, por lo que quedaron indefinidas. Con respecto a la selección en 58 titulares hubo una coincidencia total entre los tres jueces, en 43 fue parcial y, como se mencionó anteriormente, en diez no existió coincidencia.

De los tres medios con más titulares sobre gratuidad en educación, Emol contó con una leve ventaja en noticias negativas (ocho y siete respectivamente); llama la atención su alta presencia de indefinidos (cuatro), con lo que casi alcanzan la mitad de todos los titulares caracterizados bajo este parámetro. En cambio, La Tercera cuenta con una amplia proporción de encabezados negativos (11), que duplican a las positivas (cinco). Por su parte, T13 presenta una mayor presencia de titulares positivos (seis), frente a cuatro neutras, una indefinida y solamente una negativa. Desde otra lectura, estos tres medios tienen $70 \%$ de los titulares consignados como indefinidas.

\section{CONCLUSIONES}

La minería de datos no nos puede hacer pensar que estamos frente a una verdad objetiva en el análisis de la prensa, pero sí nos refleja claras tendencias acerca el comportamiento de los medios sobre algunos temas, en especial los que generan polémica y distintas visiones discordantes en la sociedad, como lo fue la gratuidad en la educación en la sociedad chilena.

Desde nuestro punto de vista, este estudio logró demostrar una visión muy compartida por muchos estudiosos y analistas de la realidad sociopolítica chilena: el descrédito de la prensa hacia el proyecto de gratuidad en la educación y, en general, hacia todo el gobierno de Michelle Bachelet. Esto no era muy difícil de prever, tomando en cuenta la clara tendencia derechista de los principales conglome- 
rados periodísticos del país que, por su línea editorial, estaban en la oposición a las reformas del gobierno de Bachelet. De hecho, el estudio de Cabalin y Antezana (2016) sostiene que Las Últimas Noticias tomó una posición crítica, preocupado por las consecuencias políticas de los cambios, defendiendo la libertad de las personas que, desde su punto de vista, se veía amenazada. La imagen más clara era la portada con un escolar en una ruleta, que indicaba que ahora el futuro de los jóvenes chilenos ya no dependía del esfuerzo de los padres, ni de las habilidades de los estudiantes, sino del azar.

Por eso, entre las diez noticias más repetidas y en el análisis aleatorio que hicimos, la calificación de los jueces fue negativa. En la misma línea se le dio una enorme difusión que recibió la asignación de gratuidad por error, y las mismas críticas al proceso por parte del senador oficialista Ignacio Walker.

En cierta medida, podemos decir que el rol crítico de la prensa remplazó a la oposición derechista, que apareció muy poco en este estudio.

Por otro lado, pese a que las voces oficialistas dominan en la información seleccionada, se ven muy pocos actores oficialistas regionales. En otras palabras, las autoridades regionales no salieron a los medios a defender la principal política pública de su gobierno. Agrava la falta el hecho que todos los gobiernos regionales cuentan con convenios con los medios, lo que les permite difundir su información.

Aunque nuestro estudio no busca establecer la influencia de los medios en la opinión pública, es interesante ver el parangón que se puede realizar entre la proporción de noticias negativas, frente al respaldo público a la gratuidad en educación que, para momentos de ese estudio era de alrededor de 39\% (Cooperativa, 2016). ${ }^{10}$

Obviamente, para otras investigaciones quedará indagar los motivos que llevaron a la prensa chilena a difundir más las noticias negativas que las positivas sobre este importante tema, que determinó la agenda política chilena durante varios ańos.

\footnotetext{
${ }^{10}$ En febrero y marzo otros sondeos hablaban de un respaldo de $41 \%$ y $45 \%$, respectivamente, pero al tratar el periodo entre marzo y julio, ésta es la encuesta más precisa, de acuerdo al periodo investigado.
} 


\section{REFERENCIAS BIBLIOGRÁFICAS}

Arrate, J. (2009). Programa de Gobierno Jorge Arrate. Recuperado de https://es.scribd.com/document/20707327/Jorge-Arrate-Programade-Gobierno.

Bachelet, M. (2013). Programa de Gobierno Michelle Bachelet. Recuperado de https://es.scribd.com/doc/179662564/Programa-Presidencial-Michelle-Bachelet-2014-2018.

Baeza-Yates, R. (2009). Tendencias en minería de datos de la Web. El profesional de la información, 18(1) 5-10.

Bellei, C., Cabalin, C., y Orellana, V. (2014). The 2011 Chilean student movement against neoliberal educational policies. Studies in Higher Education, 39(3), 426-440.

Best, M., y Meng, A. (2015). Twitter Democracy: Policy versus identity politics in three emerging African democracies. Proceedings of the Seventh International Conference on Information and Communication Technologies and Development (ACM).

Browne, R., Monsalve, S., y Romero, P. (2015). La cobertura regional del movimiento estudiantil chileno 2011: prensa impresa y prensa digital en La Región de Los Ríos (Chile). Estudios sobre el Mensaje Periodístico, 21(2), 723-740.

Cabalin, C. (2012). Neoliberal Education and Student Movements in Chile: inequalities and malaise. Policy Futures in Education, 10(2), 219-228.

Cabalin, C. (2013). Framing y Políticas Educacionales: los medios como actores políticos en educación. Estudios sobre el Mensaje Periodístico, 19(2), 635-647.

Cabalin, C., y Antezana, L. (2016). La educación en portada: la visualización de la política educacional en la prensa. Cuadernos Info., (39), 195-207.

Claude, M. (2013). Programa de Gobierno Marcel Claude. Recuperado de https://es.scribd.com/doc/179671346/Programa-Presidencial-Marcel-Claude-2014-2018.

Cooperativa.cl (2013). Bachelet: Es regresivo que quienes pueden pagar la educación no paguen. Recuperado de https://www.cooperativa.cl/ noticias/pais/michelle-bachelet/bachelet-es-regresivo-que-quienespueden-pagar-la-educacion-no-paguen/2013-04-08/132353.html 
Cooperativa.cl (2016). Adimark: Apoyo a reforma educacional baja 10 puntos y llega a 39 por ciento. Recuperado de https://www.cooperativa. $\mathrm{cl} /$ noticias/pais/politica/encuestas/adimark-apoyo-a-reforma-educacional-baja-10-puntos-y-llega-a-39-por-ciento/2016-07-06/094827. html.

Emol. (2011). Presidente Piñera afirma que la educación "es un bien de consumo". Recuperado de http://www.emol.com/noticias/nacional/2011/07/19/493428/presidente-pinera-afirma-que-la-educaciones-un-bien-de-consumo.html.

Enríquez-Ominami, M. (2009). Programa de Gobierno Marco EnríquezOminami. Recuperado dehttps://es.scribd.com/document/23239688/ Programa-de-Gobierno-Marco-Enriquez-Ominami.

Enríquez-Ominami, M. (2013). Programa de Gobierno Marco Enríquez-Ominami. Recuperado de https://es.scribd.com/document/179665104/Programa-Presidencial-Marco-Enriquez-Ominami-2014-2018.

Frei, E. (2009). Programa de Gobierno Eduardo Frei. Recuperado de https:// es.scribd.com/doc/23985909/Programa-de-Gobierno-Eduardo-Frei.

Gómez, J. (2012). Estado, dominación, hegemonía y crisis política en la sociedad neoliberal, Chile 1973-2012. En M. Thwaites (ed.), El Estado en América Latina: continuidades y rupturas (pp. 189-226). Santiago: Arcis.

Harvey, D. (2007). Breve Historia del Neoliberalismo. Madrid: Akal.

Hobsbawm, E. (1999). Historia del siglo XX. Buenos Aires: Crítica.

Instituto Nacional de la Juventud (INJUV) (2010). Gta Encuesta Nacional de la Juventud. Santiago, Chile: INJUV. Recuperado de https://www. academia.edu/3211698/6ta_Encuesta_Nacional_de_Juventud

Israel, R. (2013). Programa de Gobierno Ricardo Israel. Recuperado de http://ricardoisraelpresidente2014.blogspot.cl/p/programa-de-gobierno.html.

Jocelyn-Holt, T. (2013). Compromisos programáticos, Tomas Jocelyn-Holt. Recuperado de https://es.scribd.com/document/179690381/Compromisos-Programaticos-Tomas-Jocelyn-Holt.

Krippendorff, K. (2004). Content analysis: An introduction to its methodo$\log y$. Thousand Oaks, California: Sage.

López-García, X., Cruz-Negreira, M., y Rodríguez-Vásquez, A. (2016). Cibermedios hiperlocales ibéricos: El nacimiento de una nueva red de proximidad. Cuadernos.info, (39), 225-240. 
Matthei, E. (2013). Programa de Gobierno Evelyn Matthei. Recuperado de https://es.scribd.com/document/179657979/Programa-PresidencialEvelyn-Matthei-2013.

Miranda, R. (2013). Programa de Gobierno Roxana Miranda. Recuperado de https://es.scribd.com/document/182639738/Programa-de-Gobierno-Roxana-Miranda-2014-2018.

Moulian, T. (1997). Chile actual: anatomía de un mito. Santiago: LOM, Arcis.

Organización de las Naciones Unidas para la Infancia (UNICEF) (2014). La voz del movimiento estudiantil. Santiago: UNICEF.

Organización para la Cooperación y el Desarrollo (OCDE) (2009). La Educación superior en Chile. París: OCDE, Banco Mundial.

Parisi, F. (2013). Programa de Gobierno Franco Parisi. Recuperado de https://es.scribd.com/document/182650178/Programa-de-Gobierno-Franco-Parisi-2014-2018.

Piñera, S. (2009). Programa de Gobierno de Sebastián Piñera. Recuperado de https://es.scribd.com/document/23239960/Programa-de-Gobierno-Sebastian-Pinera-2009.

Romero, P. (2013). Análisis crítico de la representación informativa de Camila Vallejo y el Movimiento Estudiantil chileno 2011 en el diario Las Últimas Noticias. Estudios sobre el Mensaje Periodístico, 19(2) 871-888.

Saéz-Trumper, D., Castillo, C., y Lalmas, M. (2013). Social media news communities: gatekeeping, coverage, and statement bias. Proceedings of the 22nd ACM international conference on Conference on information \& knowledge management, ACM, San Francisco, California.

Scherman, A., Arriagada, A., y Valenzuela, S. (2012). La protesta en la era de las redes sociales: el caso chileno. En A. Arriagada y P. Navia (eds.), Intermedios. Medios de comunicación y democracia en Chile (pp. 181-199). Santiago: Universidad Diego Portales. Recuperado de http://cip.udp.cl/medios/ wp-content/uploads/2016/01/Capitulo_8Scherman_Arriagada_y_Valenzuela.

Sfeir, A. (2013). Programa de Gobierno Alfredo Sfeir. Recuperado de https:// es.scribd.com/document/179686051/Programa-Presidencial-AlfredoSfeir-2014-2018.

Tironi, E. (2011). ¿Por qué no me quieren? Del Piñera way a la rebelión de los estudiantes. Santiago: Uqbar Editores. 